\title{
GERD Pathophysiology, Diagnosis, Management and Treatment with a Focus on Speech Therapeutics
}

\section{Shmuel Golfeyz* MD and Aviva Bamshad CCC-SLP}

Department of Gastroenterology, Maimonides Medical Center, USA

*Corresponding Author: Shmuel Golfeyz, MD, Department of Gastroenterology, Maimonides Medical Center, USA.
Received: February 16, 2021

Published: March 16, 2021

(C) All rights are reserved by Shmuel Golfeyz

MD and Aviva Bamshad CCC-SLP.

\begin{abstract}
Gastroesophageal reflux disease (GERD) is one of the most commonly encountered diseases worldwide. Symptoms vary widely among patients and can lead to decreased quality of life. Though much has been published regarding medical GERD treatment, there is a paucity of literature on the topic of speech therapeutics as an alternative, or in addition to, standard treatment options. This review article focuses on highlighting current literature on this subject matter and providing a thorough overview of GERD. Further research and study into the utility of speech therapeutics in the treatment of GERD is necessary to help determine which therapeutic techniques are the most effective treatment options.
\end{abstract}

Keywords: GERD; Reflux; Voice; Dysphonia; Speech Language Pathology; SLP

\section{Introduction}

Gastroesophageal reflux disease (GERD) is one of the most common diseases encountered by both gastroenterologists and primary care physicians worldwide. Its prevalence in the United States (US) is estimated to be between 18.1-27.8\% [1]. Approximately $20 \%$ of the US population experiences GERD symptoms weekly [2]. Patients present with a variety of symptoms, many of which lead to a reduced quality of life [3]. One systematic review [4] found that patients with disruptive GERD symptoms had decreased work productivity and increased time off from work. These patients were also found to have decreased physical functioning and low scores on sleep scales [4]. Notably, it appears that nocturnal GERD symptoms have a greater impact on quality of life than daytime symptoms do [5]. GERD can cause a myriad of vocal disorders and standard medical therapies may not be enough to treat these.

\section{Definition}

Many different terms have been used to describe GERD. It is therefore imperative that clinicians understand the appropriate definition of a symptom, so they can accurately differentiate between diseases. Gastroesophageal reflux disease is defined as symptoms or complications which result from reflux of gastric contents into the esophagus, oral cavity, or lungs [3]. Heartburn is a common symptom of GERD and is defined as a retrosternal burning sensation which can sometimes be accompanied by a bitter taste in the mouth. Dyspepsia is defined as an upper abdominal discomfort which is associated with nausea, belching, and bloating and can be associated with food intake. Dyspeptic symptoms can be due to GERD and other gastrointestinal pathologies. GERD is classified as either: symptoms without erosive findings on endoscopy (nonerosive reflux disease or NERD); or symptoms with erosive findings (erosive reflux disease or ERD) [3]. 
Pathophysiology

The pathophysiology of GERD is complex. It is believed to be caused by several different mechanisms in combination with specific underlying medical conditions. GERD has been associated with causes such as pregnancy, esophagitis, scleroderma, ascites, obesity, medications, and foods such as alcohol, chocolate, coffee, fatty foods, orange juice, peppermint, and tomatoes [6]. The major underlying mechanistic cause of GERD is thought to be due to weakness in the lower esophageal sphincter which allows for reflux of gastric contents into the esophagus. The combination of a structural deficit, and certain medical conditions, predispose patients to having higher incidences of GERD. For example, GERD is prevalent in obese patients due to a weakness at the lower esophageal sphincter and reflux of gastric contents from high intrabdominal compartment pressure from the obesity [3].

Importantly, due to reflux of gastric contents into the laryngopharynx and oropharynx, many vocal complications arise including chronic laryngitis and other voice disorders. Chronic laryngitis develops from acidic gastric contents refluxing onto the vocal folds, which leads to swelling and narrowing of the vocal passage. This damage, over time, places a significant strain on the vocal system and decreases patients' quality of life.

\section{Symptoms}

Heartburn and regurgitation have high specificity but low sensitivity, and are the most reliable symptoms to diagnose GERD based on history alone [3,7]. Dyspepsia is also a common symptom that can represent a variety of underlying medical conditions and many patients with dyspepsia are subsequently diagnosed with GERD. Additionally, dyspeptic symptoms such as early satiety, belching, bloating, and epigastric pain tend to respond well to proton pump inhibitor (PPI) therapy [3]. GERD symptoms are common after meals and with recumbency. Alarm symptoms, which raise concern for serious underlying pathology and warrant further evaluation with upper gastrointestinal endoscopy, include dysphagia (difficulty swallowing), odynophagia (pain with swallowing), weight loss, microcytic anemia, new symptoms in a patient with age greater than 60 years, and symptoms lasting more than 5 years $[3,8]$.

\section{Differential diagnosis and complications}

Many GERD symptoms tend to overlap with other gastrointestinal diseases or even other organ systems. It is therefore imperative for clinicians to evaluate symptoms appropriately and rule out other potentially serious pathologies. These include but are not limited to, acute coronary syndrome, pancreatitis, cholecystitis, esophageal dysmotility, esophageal spasm, scleroderma, and eosinophilic esophagitis. The differential diagnosis for gastrointestinal causes of GERD includes achalasia, esophageal or stomach cancer, peptic ulcer disease, functional dyspepsia, gastritis, and gastroparesis [7].

Complications from GERD include but are not limited to, erosive esophagitis, Barrett's esophagus, peptic stricture, esophageal adenocarcinoma, dysphagia, pulmonary disease, dental erosions, chronic laryngitis, and other voice disorders $[3,7]$.

\section{Work-up and diagnosis}

The first step in evaluating patients with symptoms of GERD is to determine whether or not the patient needs to undergo further diagnostic testing. It is important for clinicians to have a high index of suspicion of other causes, such as cardiac or pulmonary etiologies. Diagnostic testing is performed to avert misdiagnosis, to identify complications, and to evaluate treatment failures [6]. Once more potentially concerning causes of GERD symptoms have been ruled out, clinicians can focus on diagnosis and management.

GERD is diagnosed by obtaining a history and performing a physical exam. Patients presenting with typical symptoms and no red flags can be treated empirically without further diagnostic workup. A diagnosis of GERD can be also be considered in patients with chronic cough, asthma or laryngitis, however, careful evaluation for non-GERD causes should be explored in such cases [3]. For those in whom the diagnosis is unclear, unfortunately, there are no perfect tests to help clinicians verify a diagnosis. The exact definition of symptoms as well as location and perceived burdensomeness vary widely among patients and providers, which makes the diagnosis that much more challenging. Additionally, as previously discussed, GERD symptoms overlap with many other pathologies skewing the diagnosis further.

Due to symptom variability, researchers and clinicians have been focusing on developing standardized questionnaires to help aid clinicians to appropriately diagnose GERD. Initial questionnaires included the GERD Impact Scale and the ReQuest questionnaire. Unfortunately, these questionnaires were found to be too long, complicated and not validated for clinical care [9]. A recently developed questionnaire known as the GerdQ is standardized and 
has been validated for use in the clinical setting [9]. Patients are asked six questions and told to score the frequency of their symptoms over the previous week. Four questions focus on symptoms and two questions focus on disease impact. A symptom score $<8$ suggests a low probability of GERD and $\geq 8$ suggests GERD. A score $\geq 8$, with a combined score on the two disease impact questions of $>3$, suggest GERD with a high impact on quality of life.

\section{Diagnostic modalities}

\section{Endoscopy}

Endoscopy is the test of choice for patients who need to undergo further diagnostic testing. This testing modality is typically reserved for patients with red flags or longstanding GERD symptoms. It allows for direct visualization of the mucosa and allows the operator to collect biopsies for tissue analysis. Endoscopy can also be used to evaluate for complications of GERD including esophagitis, strictures, ulcers, erosions, and Barrett's esophagus (metaplasia of the esophageal mucosa) [7].

\section{Esophageal $\mathrm{pH}$ or $\mathrm{pH}$-impedance Testing}

Esophageal $\mathrm{pH}$ or $\mathrm{pH}$-impedance testing is another testing modality which is performed for some patients. Monitoring of $\mathrm{pH}$ levels is performed in the ambulatory setting, allowing for determination of reflux frequency, esophageal acid exposure, and association between symptoms and reflux $[3,7]$. Testing is performed by placing a capsule endoscopically or via a transnasal catheter. $\mathrm{pH}$-impedance testing has very high sensitivity (77-100\%) and specificity (85-100\%), specifically in patients with erosive esophagitis [3].

Other lesser used testing modalities include barium radiography and manometry. Barium radiography can demonstrate esophageal strictures or masses but is not used to diagnose GERD. Esophageal manometry can also be used in some instances to look for esophageal dysmotility.

\section{Laryngoscopy}

Patients with voice symptoms such as laryngitis should be referred to an otolaryngologist to rule out causes other than GERD. Direct visual laryngoscopy can show edema and erythema of the larynx which can suggest reflux-induced laryngitis. However, it is not recommended to diagnose reflux-induced laryngitis from laryngoscopy alone, as one prospective study demonstrated laryngeal irritation in over $80 \%$ of healthy controls [10]. Another study found a lot of intra and inter-observer variability, intra-rater variability, and very low concordance when five otolaryngologists blindly evaluated 120 video laryngoscopies [11]. Furthermore, there are many non-GERD causes of laryngeal irritation and thus a definitive diagnosis of reflux laryngitis cannot be made by use of laryngoscopy alone [3].

\section{Treatment and management}

\section{Lifestyle modification}

Lifestyle modification is one of the mainstays of GERD treatment. Cessation of alcohol, tobacco, and trigger foods such as caffeine, coffee, chocolate, spicy foods, acidic food, and food with high-fat content, is advised. Other interventions include weight loss, post-prandial head of the bed elevation, and avoidance of latenight meals (i.e. meals 2-3 hours prior to bedtime) [3,7].

A 2006 systematic review of 16 trials concluded that weight loss and elevation of the head of the bed were the most effective lifestyle changes which improved esophageal pH and GERD symptoms [12]. Another study evaluating reflux disease recurrence, defined by recurrence of symptoms requiring more medications after 4-8 weeks of prior PPI treatment, found that patients who slept within 3 hours of dinner had a much higher chance of having recurrent symptoms [13].

\section{Medication treatment}

\section{Proton pump inhibitors}

Proton pump inhibitors (PPIs) are a major class of medications used to inhibit acid secretion and considered the mainstay of medical treatment. The PPI is initially a prodrug which gets activated by gastric acid. The drug then covalently binds to the parietal $\mathrm{H}+, \mathrm{K}+-$ ATPase pump and inhibits hydrogen chloride secretion, thereby increasing the $\mathrm{pH}$ of the stomach. The presence of acid secretion is necessary for PPIs to work (due to the prodrug state) thus it is recommended that PPIs be given approximately 30 minutes before breakfast to ensure that the pumps are active when peak concentrations of the PPIs are present in the blood [14]. PPIs have been associated with nearly twice as fast healing rates when compared with histamine 2 receptor antagonists (H2RAs) and placebos in treating erosive esophagitis $[15,16]$. It is reasonable to treat patients with extraesophageal manifestations such as asthma, chronic cough, and laryngitis (in the setting of typical symptoms and signs of GERD) with a PPI [3]. However, one meta-analysis found that PPI therapy had no significant advantage over placebo in improving symptoms for suspected GERD-related chronic laryngitis [17]. 
Adverse effects of proton pump inhibitors

Many adverse effects of long-term PPI use are now being recognized. Gastroenterologists, as well as general practitioners, are paying much closer attention to patients on long-term PPI therapy. Adverse effects include vitamin B12 deficiency, pneumonia, hip fractures, osteoporosis, iron deficiency anemia, clostridium difficile diarrhea, and even chronic kidney disease. Though there is disagreement regarding if indeed long-term PPI use leads to all of the aforementioned adverse effects, when avoidable it is best not to keep patients on PPIs for extended periods of time [3].

\section{Histamine 2 receptor antagonists}

In addition to PPIs, histamine 2 receptor antagonists are used to treat GERD. H2RAs work by binding to and blocking the $\mathrm{H} 2$ receptors on the parietal cells. Normally, histamine binds to these receptors and causes elevation of intracellular cyclic AMP concentrations and activation of protein kinase A (PKA). PKA causes phosphorylation of cytoskeletal proteins involved in the transport of the gastric $\mathrm{H}+, \mathrm{K}+$-ATPase from the cytoplasm to the plasma membrane. By blocking this pathway, gastric acid secretion is inhibited [14]. Therefore, a bedtime H2RA is added to PPI therapy in patients with refractory symptoms to help suppress night-time acid production [3].

\section{Baclofen}

Baclofen, a GABA b agonist is an additional treatment option which works by decreasing transient LES relaxation [18].

\section{Potassium competitive acid blockers}

Potassium competitive acid blockers (P-CABs) are a new class of acid neutralizing medications which have recently been developed and are being widely used in Japan [19]. One P-CAB which has been studied is Vonoprazan. It provides more potent, faster, and steadier acid suppression as compared with PPIs. Vonoprazan is more stable in the parietal call secretory canaliculi, has a longer half-life, has a lower half-maximal inhibitory concentration for inhibition of proton pump activity, and is resistant to CYP2C19 degradation. Within 4 hours of administration, gastric $\mathrm{pH}$ has been documented to rise above 7 and has remained above 5 for 24 hours. Vonoprazan has shown better results in treating some high-grade PPI resistant reflux esophagitis. The negative aspects of P-CABs are that they are likely to have similar adverse effects as PPIs due to their ability to provide strong acid suppression. The evidence to support this has not yet been established. Long-term studies and post-market surveillance will be imperative in monitoring for adverse events. A future study that focuses on symptom resolution will also be needed to determine the clinical impact of P-CABs [19].

\section{Surgical treatment}

Aside from medical management, there are surgical treatment options for patients with refractory symptoms. These include bariatric surgery (for obese patients) and laparoscopic fundoplication. Surgical treatment is usually reserved for patients with poor medication compliance, adverse effects from medications, desire to discontinue medical therapy, refractory esophagitis, large hiatal hernia, or persistent symptoms $[3,7]$. The highest surgical responses are noted in patients with typical symptoms of heartburn and/or regurgitation which demonstrated a good response to PPIs. Unfortunately, surgical treatment is not without its risks, the most concerning of which is an increased short-term risk of mortality. Most commonly though, patients develop gas-bloat syndrome after surgery. Interestingly, outcomes for extraesophageal symptoms such as pulmonary function tests have not been established to improve after surgery [3].

\section{Other therapies}

Other therapies, include endoscopic radiofrequency augmentation of the LES, silicone injection into the LES, and suturing of the LES. These therapies have failed to demonstrate long-term efficacy [3].

\section{Treatment of refractory GERD}

In patients with refractory symptoms after initiation of lifestyle and medication treatment, the first step is to assess appropriate compliance. Is the medication dose correct? Is the medication being administered appropriately? If these parameters are acceptable, then a trial treatment with a different PPI should be attempted. If symptoms continue to persist, then the patient should be evaluated with endoscopy to exclude non-reflux esophageal causes such as eosinophilic or erosive esophagitis. If endoscopy is unrevealing, reflux monitoring should be performed to quantify reflux and assess the relationship to symptoms. Patients with a low pretest probability of GERD should be tested with $\mathrm{pH}$ or $\mathrm{pH}$ impedance tests while off medications. Patients with high pretest probability for GERD should be tested with pH-impedance while on medications. Patients with refractory atypical symptoms should 
be referred to pulmonary, otolaryngology and allergy specialists to exclude non-GERD etiologies. Standard otolaryngology assessment includes visualization of the vocal folds and possibly swallow function with fiberoptic endoscopic examination of swallowing (FEES). If workup returns abnormal, then treatment should be targeted to the underlying cause. Once non-GERD etiologies have been ruled out in patients with atypical symptoms, reflux monitoring can be performed [3].

\section{Speech language pathology role and treatment}

As with many other medical disorders, a multidisciplinary approach is necessary for optimal patient care, especially for patients with atypical GERD symptoms. Due to symptom overlap, there are times that patients will have symptoms that may not be due to actual GERD. Rotsides., et al. [20] demonstrated that pediatric patients with recalcitrant aerodigestive symptoms, $67 \%(n=37)$ of whom had been previously diagnosed with GERD, were effectively treated using a multidisciplinary aerodigestive clinic approach. Rotsides., et al. looked at presenting complaints, evaluation, management, and outcome of patients during 12 monthly visits. The patients were evaluated and seen by a multidisciplinary team including otolaryngologist, gastroenterologist, pulmonologist, nurse practitioner and speech language pathologist (SLP). Clinic evaluation included flexible nasopharyngolaryngoscopy, endoscopy, and pulmonary function testing. Testing revealed specific diagnosis including vocal cord dysfunction, laryngeal cleft, pulmonary infection, adenoid hypertrophy, reflux disease, tracheomalacia, laryngomalacia, celiac disease, cilia abnormality, helicobacter pylori, duodenal web, and eosinophilic esophagitis. Once a diagnosis was made, treatment was chosen to address the underlying problem. SLP's treated the patients with breathing and voice therapy. The multidisciplinary team approach resulted in improved to resolved symptoms in $73 \%$ of patients [20].

Therefore, in addition to otolaryngologist and pulmonologist referrals, patients displaying vocal disorders such as dysphonia or laryngitis should be referred to an SLP (after appropriate evaluation by an otolaryngologist). SLP's can provide an array of therapeutic techniques and can play a crucial role in ensuring optimal patient outcomes for GERD patients displaying vocal disorders.

While research continues regarding the best voice therapeutic techniques to treat GERD-related voice disorders, the results of multidisciplinary treatment in small studies are promising. Due to a lack of strong prospective randomized trials in this area, current techniques have displayed differing results between studies. For example, while behavioral voice therapy is recommended for the treatment of many different voice disorders, previous reviews have not shown which treatment approach is most effective. Desjardins., et al. [21] performed a systematic review analyzing fifteen studies covering five categories of voice disorders (including GERD-induced disorders) and seven specific voice therapy techniques. The therapy techniques used for GERD related voice disorders included the yawn-sigh technique, relaxation and breathing exercises, glottal fry, chant talk, chewing exercises, and humming. Although there were statistically significant improvements within the studies, the actual effectiveness of these techniques was difficult to ascertain because of the lack of standard consensus regarding what constituted a beneficial outcome measure [21].

On the opposite side, Vashani., et al. [22] evaluated whether treatment with omeprazole and voice therapy compared with omeprazole alone would improve voice quality in patients with GERD. In this study, voice therapy consisted of vocal hygiene (which focused on the reduction of laryngeal irritants), vocal rest, adequate hydration, reduction of vocal loudness and speech rate, reduction of vocal abuse and hard glottal attack, and elimination of chronic throat clearing and coughing. Additionally, they used direct techniques of voice therapy which focused on different organ systems. The musculoskeletal treatment focused on relaxation exercises in neck rotation, laryngeal massages, yawn-sigh, and chewing therapies. The respiratory system focused on breathing exercises. The somatosensory system focused on humming and glottal fry. The outcome analysis focused on four acoustic parameters (jitter, shimmer, normalized noise energy, and harmonic-to-noise ratio) and two perceptual parameters (hoarseness and breathiness). The study found that omeprazole and voice therapy generated better vocal outcomes than omeprazole alone. The study did have some limitations though, including, a small sample size, laryngoscopy not being repeated after therapy, and being unable to compare voice changes in various grades of GERD [22].

Despite the lack of consistent or comparable data, SLP's play an important role in the overall assessment, monitoring, and treatment of vocal disorders in patients with GERD. The SLP's role should be to take a detailed history of the patient's diet, insulting 
factors (such as voice overuse), and determine the time lapse between eating and reclining. SLP's should monitor hoarseness and help align vocal bio-mechanics. Additionally, SLP's should counsel patients on appropriate medication administration, avoidance of trigger foods, elevation of the head of the bed, weight loss exercises, and avoidance of heavy meals within 3 hours of a performance or intense vocal activity. Although this can be redundant (as the gastroenterologist or primary care physician should have already counseled the patient on this) having an SLP reiterate and reinforce proper behaviors is essential in providing effective patient care. After counseling, SLP's can treat patients with voice therapy techniques.

\section{Chronic cough SLP treatment}

Chronic cough is the most common symptom experienced by patients with chronic GERD. Several studies have looked into the utility of speech therapy as a treatment option for chronic cough [23-26]. One study - a randomized single-blind placebo-controlled trial of 87 patients - found a significant reduction in breathing, voice, upper airway, and cough symptoms after intervention with an SLP. The study demonstrated improvements in other areas such as auditory perceptual ratings of vocal quality (breathy, rough, strain and glottal fry) and significant improvement in vocal acoustic parameters (maximum phonation time, jitter, and harmonicto-noise ratio) [23]. The first step in the treatment of cough is for the SLP to perform an assessment looking at cough characteristics, urge to cough, laryngeal assessment, and voice symptoms. This is followed by training using several tools including education, cough suppression strategies, vocal hygiene training, and psycho-educational counselling [27]. SLP's should teach patients what causes a person to cough, the unnecessary nature of the cough, and the consequences of chronic coughing such as laryngeal irritation or trauma. It should be stressed that patients have the ability to control their cough if they use the right techniques. Patients should be taught to identify precipitating sensations of a cough and to substitute the urge to cough with another response such as swallowing. In order to decreases laryngeal irritation, vocal hygiene techniques are also taught. This includes teaching to avoid mouth breathing, keeping the mouth adequately hydrated, and to quit smoking [26].

\section{Dysphonia SLP treatment}

Dysphonia is another common symptom experienced by GERD patients. It is defined as an alteration in vocal quality, loudness, or pitch, which impairs communication and/or quality of life. Dysphonia clinical practice guidelines [28] strongly advocate clinicians to refer patients to voice therapy if the cause of dysphonia is amenable to therapy. Clinicians should either perform or refer for diagnostic laryngoscopy before prescribing voice therapy and the results should be communicated with the SLP to help guide an individualized treatment plan. If a patient has GERD symptoms and dysphonia, it is important that clinicians not prescribe antireflux medications to treat the dysphonia without first visualizing the larynx with laryngoscopy [28]. This is so clinicians do not miss another more concerning cause of the dysphonia (such as cancer).

\section{Diaphragmatic breathing exercises}

Diaphragmatic breathing exercises as a treatment for patients with PPI refractory GERD and belching has also been demonstrated by Ong., et al. [29] This prospective study considered 36 patients who had PPI refractory GERD and a belching visual analogue scale (VAS) score of 6 or more. Fifteen of the patients were started on a standardized diaphragmatic breathing exercise protocol and the other twenty-one were control subjects, but near the end of the study were also treated. Subjects were evaluated with questionnaires before, during, and after treatment. The primary outcome looked at reduction in belching VAS score by $50 \%$ or more after treatment. Secondary outcomes included GERD symptoms (evaluated using the reflux disease questionnaire) and quality of life scores (evaluated using the Reflux-Qual Short Form and EuroQoLVAS).

The exercise protocol performed in this study consisted of having patients lay in the supine position with one hand on the chest and the other on the upper abdomen. The patient was then instructed to inhale through the nose and exhale with the mouth open wide, moving only the abdomen. The subject had to completely inflate and deflate the abdomen with each breath while keeping the chest and shoulders still. Inhalation was performed slowly by the subject counting internally to 4 and then maintaining the breath for an additional 4 counts. The subject then exhaled slowly and gradually from 4 to 8 counts. If the subject had difficulty prolonging exhalation, s/he was instructed to breathe through pursed lips instead. These exercises were progressively taught to be performed while sitting and then standing. The patient was instructed to practice this at home by performing 30 breaths for 5 minutes 3 times daily, 
and for 5 minutes when symptomatic. In the study, patients were seen for either 4 sessions, or until the SLP decided the patient was independent in both supine and upright positions. This exercise technique is relatively simple to teach. In combination with other therapeutic techniques, the study showed significantly improved symptoms and quality of life for patients receiving the treatment.

The results showed that 9 of the 15 patients $(60 \%)$ in the treatment group achieved the primary outcome, whereas none of the patients in the control group did. Eighty percent of patients in the treatment group significantly reduced belching frequency compared with $19 \%$ of the control subjects. Symptoms of GERD were also significantly decreased as measured by a decrease in the mean reflux disease questionnaire. Quality of life scores were also increased in the treatment group compared with controls. These changes were still sustained at 4 months after treatment (which is when they distributed the final questionnaire). After the 21 control group patients were treated, another 11 (52\%) patients achieved the primary outcome.

\section{Conclusion}

GERD is a complex disease which impacts patients in many ways. Standard therapies are aimed at first treating patients by using lifestyle modifications and then medical therapy. For patients with atypical symptoms, multidisciplinary care is paramount for effective treatment. SLP's play a big role in treating GERD-induced voice disorders. Although there is a lack of strong evidence to support specific speech therapies, most small studies in the literature show a statistically significant increase in quality of life and other symptoms when an SLP is used in conjunction with standard medical therapy. Although there is no perfect approach as of yet, hopefully this article has highlighted some of the techniques that have been used and shown to have some benefit. SLP's can utilize whichever therapies they find appropriate and create individualized therapy plans for each patient. Much more research is needed on this topic and researchers need to perform large prospective randomized controlled trials to help answer the question of which vocal therapeutic option or options are the best in treating specific voice disorders. Hopefully, by doing so, patients will ultimately reap the benefit.

The authors would like to acknowledge Gabriel R. Aaronson for his help in proofreading and critically appraising the article.

\section{Bibliography}

1. El-Serag HB., et al. "Update on the epidemiology of gastrooesophageal reflux disease: a systematic review". Gut 63.6 (2014): 871-880.

2. Locke GR., et al. "Prevalence and clinical spectrum of gastroesophageal reflux: a population-based study in Olmsted County, Minnesota". Gastroenterology 112.5 (1997): 1448-1456.

3. Katz PO., et al. "Guidelines for the diagnosis and management of gastroesophageal reflux disease". The American Journal of Gastroenterology 108.3 (2013): 308-328.

4. Becher A and El-Serag H. "Systematic review: the association between symptomatic response to proton pump inhibitors and health-related quality of life in patients with gastro-oesophageal reflux disease". Alimentary Pharmacology and Therapeutics 34.6 (2011): 618-627.

5. Gerson LB and Fass R. "A systematic review of the definitions, prevalence, and response to treatment of nocturnal gastroesophageal reflux disease". Clinical Gastroenterology and Hepatology : The Official Clinical Practice Journal of the American Gastroenterological Association 7.4 (2009): 372-378.

6. Kahrilas PJ. "Clinical practice. Gastroesophageal reflux disease". The New England Journal of Medicine 359.16 (2008): 1700-1707.

7. Badillo R and Francis D. "Diagnosis and treatment of gastroesophageal reflux disease". World Journal of Gastrointestinal Pharmacology and Therapeutics 5.3 (2014): 105-112.

8. DeVault KR and Castell DO. "Updated guidelines for the diagnosis and treatment of gastroesophageal reflux disease". The American Journal of Gastroenterology 100.1 (2005): 190-200.

9. Jonasson C., et al. "Validation of the GerdQ questionnaire for the diagnosis of gastro-oesophageal reflux disease". Alimentary Pharmacology and Therapeutics 37.5 (2013): 564-572.

10. Milstein CF., et al. "Prevalence of laryngeal irritation signs associated with reflux in asymptomatic volunteers: impact of endoscopic technique (rigid vs. flexible laryngoscope)". The Laryngoscope 115.12 (2005): 2256-2261.

11. Branski RC., et al. "The reliability of the assessment of endoscopic laryngeal findings associated with laryngopharyngeal reflux disease". The Laryngoscope 112.6 (2002): 1019-1024. 
12. Kaltenbach T., et al. "Are lifestyle measures effective in patients with gastroesophageal reflux disease? An evidence-based approach". Archives of Internal Medicine 166.9 (2006): 965-971.

13. Yang JH., et al. "Recurrence of gastroesophageal reflux disease correlated with a short dinner-to-bedtime interval". Journal of Gastroenterology and Hepatology 29.4 (2014): 730-735.

14. Shin JM., et al. "Pharmacokinetics and pharmacodynamics of the proton pump inhibitors". Journal of Neurogastroenterology and Motility 19.1 (2013): 25-35.

15. Labenz J and Malfertheiner P. "Treatment of uncomplicated reflux disease”. World Journal of Gastroenterology 11.28 (2003): 4291-4299.

16. Chiba N., et al. "Speed of healing and symptom relief in grade II to IV gastroesophageal reflux disease: a meta-analysis". Gastroenterology 112.6 (1997): 1798-1810.

17. Qadeer MA., et al. "Proton pump inhibitor therapy for suspected GERD-related chronic laryngitis: a meta-analysis of randomized controlled trials". The American Journal of Gastroenterology 101.11 (2006): 2646-2654.

18. Koek GH., et al. "Effect of the GABA (B) agonist baclofen in patients with symptoms and duodeno-gastro-oesophageal reflux refractory to proton pump inhibitors". Gut 52.10 (2003): 13971402.

19. Kinoshita Y., et al. "Management of GERD: Are Potassium-Competitive Acid Blockers Superior to Proton Pump Inhibitors?" The American Journal of Gastroenterology 113.10 (2018): 1417-1419.

20. Rotsides JM., et al. "Is a Multidisciplinary Aerodigestive Clinic More Effective at Treating Recalcitrant Aerodigestive Complaints Than a Single Specialist?" The Annals of Otology, Rhinology, and Laryngology 126.7 (2017): 537-543.

21. Desjardins M., et al. "A Systematic Review of Voice Therapy: What "Effectiveness" Really Implies". Journal of Voice : Official Journal of the Voice Foundation 31.3 (2017): 392.e13-392.e32.

22. Vashani K., et al. "Effectiveness of voice therapy in reflux-related voice disorders". Diseases of the Esophagus : Official Journal of the International Society for Diseases of the Esophagus 23.1 (2010): 27-32.
23. Vertigan AE., et al. "Efficacy of speech pathology management for chronic cough: a randomised placebo controlled trial of treatment efficacy". Thorax 61.12 (2006): 1065-1069.

24. Murry T., et al. "Respiratory retraining of refractory cough and laryngopharyngeal reflux in patients with paradoxical vocal fold movement disorder". The Laryngoscope 114.8 (2004): 1341-1345.

25. Blager FB., et al. "Voice therapy techniques adapted to treatment of habit cough: a pilot study". Journal of Communication Disorders 21.5 (1988): 393-400.

26. Gibson PG and Vertigan AE. "Speech pathology for chronic cough: a new approach". Pulmonary Pharmacology and Therapeutics 22.2 (2009): 159-162.

27. Perotin JM., et al. "Managing patients with chronic cough: challenges and solutions". Therapeutics and Clinical Risk Management 14 (2018): 1041-1051.

28. Stachler RJ., et al. "Clinical Practice Guideline: Hoarseness (Dysphonia) (Update)". Otolaryngology--Head and Neck Surgery : Official Journal of American Academy of OtolaryngologyHead and Neck Surgery 158 (2018): S1-s42.

29. Ong AM., et al. "Diaphragmatic Breathing Reduces Belching and Proton Pump Inhibitor Refractory Gastroesophageal Reflux Symptoms". Clinical Gastroenterology and Hepatology: The Official Clinical Practice Journal of the American Gastroenterological Association 16.3 (2018): 407-416.e2.

\section{Assets from publication with us}

- Prompt Acknowledgement after receiving the article

- Thorough Double blinded peer review

- Rapid Publication

- Issue of Publication Certificate

- High visibility of your Published work

Website: www.actascientific.com/

Submit Article: www.actascientific.com/submission.php

Email us: editor@actascientific.com

Contact us: +919182824667 